\title{
L'évolution des profils des acteurs de la politique de la ville
}

Par Vanessa Girard

La politique de la ville est mise en place au début des années 1980 d'après deux nouveautés : d'une part, il s'agit de ne plus traiter individuellement les problèmes sociaux comme cela avait été le cas jusque là puisque les quartiers regroupent une population souffrant des mêmes difficultés, et, ce sont les communes qui doivent être au premier plan dans la gestion de cette nouvelle politique. Cette politique ne traite donc plus de l'individu mais du territoire, d'où le nom de développement social « urbain ». D'autre part, au fil du temps, les problèmes relevant tant de l'éducation que de la justice ou du développement économique sont intégrés à une seule et même démarche comme dans le contrat de ville ou l'actuel CUCS (Contrat urbain de cohésion sociale) qui est composé de cinq axes au niveau national, à savoir : cadre de vie et habitat, emploi et développement économique, réussite éducative, citoyenneté et prévention de la délinquance et pour finir santé. Les compétences relevant originellement de divers ministères sont regroupées et les démarches partenariales, très vivement encouragées, se multiplient car elles sont nécessaires à la bonne mise en œuvre d'actions aussi diverses que variées.

L'émergence de la politique de la ville s'accompagne de la création de ce que l'on nomme «les nouveaux métiers ». En effet, la politique de la ville fait émerger des métiers atypiques. Cette caractéristique, évidente dès le départ, vaut encore actuellement avec une difficulté toujours présente à professionnaliser ces nouveaux métiers comme nous le verrons par la suite. Des postes de "chef de projet», "agent de développement», "chargé de mission politique de la ville » ou « médiateurs »sont ouverts dans les communes, financés en grande partie par 1'Etat. L'une des premières difficultés qui apparaît est de trouver des personnes souhaitant agir dans le cadre de la politique de la ville et ayant les qualités nécessaires.

\section{De l’animateur socioculturel au diplômé de Sciences Po}

Comme 1'analyse Jacques Ion ${ }^{1}$, les premiers professionnels à répondre favorablement à la politique de la ville sont généralement d'anciens animateurs socioculturels, éducateurs spécialisés et travailleurs sociaux en milieu ouvert, fortement impliqués dans le développement social. Il s'agit majoritairement d'hommes, âgés de 30 à 45 ans, ayant ou ayant eu une forte activité militante soit dans la sphère politique, soit dans le monde associatif. Ils sont en effet les premiers à s'impliquer dès l'annonce de la nouvelle politique sociale qui prend désormais attache à un territoire et non à une catégorie de personnes et qui laisse place à une transversalité entre les différents domaines d'application. Contrairement à ce que l'on pourrait croire, ils sont très faiblement suivis par les assistantes sociales qui, elles, ne souhaitent pas s'impliquer dans une politique où l'individu ne serait plus au centre. L'enquête sur les professionnels du développement local menée par Claude Cohen en $2003^{2}$ confirme cela : $32 \%$ des professionnels interrogés ayant répondu au questionnaire (au total 57) possèdent un diplôme dans le travail social et l'animation. Ils sont plus âgés que les autres professionnels diplômés en urbanisme, géographie, développement local, économie, sciences politiques et droit puisque seulement un d'entre eux a moins de 30 ans. Deux des chargés de mission politique de la ville et du développement social urbain dans l'agglomération de Périgueux $^{3}$ sur quatre proviennent du milieu de l'animation locale. Ce sont les deux seuls à avoir plus de 35 ans et à avoir travaillé pour le compte, ou en collaboration étroite, avec les 
associations de quartier. Tous deux sont agents de la fonction publique territoriale et en poste depuis plusieurs années.

Cette évolution des profils des professionnels de la politique de la ville s'accompagne d'un changement de statut : en effet, si les contractuels étaient largement représentés au sein de la catégorie des chefs de projet ou chargés de mission jusque là, un phénomène de " municipalisation » s'est amorcé depuis le contrat de ville $2000-2006^{4}$ et se prolonge encore aujourd'hui. Trois explications peuvent être apportées pour l'expliquer; les communes ont souhaité prendre le contrôle de la politique de la ville puisqu'il s'agit d'une politique agissant exclusivement sur leur territoire et elles disposent l'un outil majeur qui est, bien évidemment, le logement social puisque les organismes dépendent des municipalités. Les acteurs de la politique de la ville, financés au départ à moitié par les communes et par 1'Etat, étaient en situation de précarité professionnelle; ils ont été conduits à s'intégrer aux services municipaux afin de faciliter leurs actions et de réduire les risques professionnels à leur endroit. D'autre part, leur relation avec les élus souffrait de 1'antithèse « élu = représentant des habitants ; chef de projet $=$ voix des populations déclassées, sans représentant ». Le chef de projet était perçu comme l'avocat des habitants non représentés par les élus. Un climat de tension a pu s'installer et freiner l'action des professionnels à certains égards. Que la municipalisation des acteurs de la politique de la ville ait un impact positif ou négatif, nous ne traiterons pas cette question ici mais nous formons cette affirmation afin de comprendre l'évolution du profil des acteurs car un lien entre les deux est perceptible. Les anciens travailleurs sociaux ont souhaité, pour la plupart, une meilleure sécurité professionnelle et les nouveaux diplômés en droit, sciences politiques cherchent à faire carrière dans l'administration.

L'une des avancées en matière de politique de la ville est bien évidemment la création, au fur et à mesure que le temps passe, des diplômes en développement local. Du master professionnel de 1'Université de Paris 1 «développement local: acteurs sociaux et dynamiques spatiales » au master de Sciences Po «administration et action publiques », les écoles et universités créent de plus en plus de diplômes de second cycle qui mènent aux carrières de chef de projet politique de la ville ou chargé de mission développement local. Un apprentissage très généraliste sert d'une part à préparer ces futurs professionnels aux concours de l'administration publique territoriale, mais aussi à être à même de gérer tous types de projets en leur donnant des connaissances nécessaires en droit public, en économie, en finances, en histoire sociale, en communication, etc. Si les premiers professionnels de la politique de la ville n'avaient pas l'ensemble de ces savoirs élémentaires, ils ont su s'adapter et apprendre, en même temps qu'ils ont « inventé » les méthodes qui sont encore aujourd'hui utilisées. Certains chargés de mission de «l'ancienne école » interrogés, à savoir les anciens animateurs socioculturels, pensent que les nouveaux diplômés leur apportent beaucoup de savoirs techniques, dont eux ne disposent pas tandis qu'ils transmettent leur expérience en matière de montage de dossier ${ }^{5}$. Bien que très différents, leurs profils ne s'opposent bien évidemment pas mais tendent même, d'après certains, à se compléter. Si l'on peut penser que la catégorie des chefs de projet anciens militants est plus active ou plus impliquée en raison de leurs activités, cela n'est pas le cas. En effet, de manière assez générale, l'implication personnelle des professionnels du développement social est globalement très importante quel que soit le profil de ceux-ci. De profils très différents, nous ne pouvons que constater l'uniformisation des méthodes employées.

Vers une professionnalisation de la politique de la ville? 
La question de la professionnalisation a été soulevée à de nombreuses reprises. Le rapport Brévan-Picard, les nombreux articles publiés par la DIV $^{6}$ dans les dossiers thématiques, les réunions de l'inter réseaux DSU sont la preuve du questionnement permanent que les professionnels de la politique de la ville portent à leur travail, à leur métier, à leur statut... Si toutes les professions n'ont pas ce genre d'interrogation, il apparaît évident que peu de «métiers » sont, dans leur exercice, différents d'une commune à l'autre ou d'une région à l'autre, tout en portant le même intitulé de poste. Si un avocat aura toujours pour mission de défendre son client en utilisant la loi, le chef de projet, lui, doit permettre de faciliter le développement d'un quartier mais selon le lieu, il sera amené à employer différents moyens et à naviguer entre différents champs: social, économique, culturel... Ainsi, il n'existe pas deux chefs de projet qui aient exactement les mêmes missions et les mêmes préoccupations au même moment. Le « flou » ${ }^{7}$ des métiers de la politique de la ville crée la nécessité pour ces professionnels de s'interroger sur leur métier. Les compétences sont aussi diverses que les connaissances demandées pour un poste. «Force de proposition, doté d'un esprit de synthèse et d'analyse. Capacité à la conduite de réunions avec des partenaires tant publics que privés. Qualités relationnelles indispensables", voilà un exemple d'offre d'emploi au poste de chef de projet ${ }^{8}$. Diverses connaissances et compétences sont ainsi demandées mais il y a une absence de critères de recrutement communs aux diverses collectivités. Il est également intéressant de noter que le terme de « qualification » est absent des discours au profit du terme de « compétences ».

En effet, les annonces décrivant les compétences nécessaires à l'exercice des fonctions de «chef de projet» varient d'une collectivité à l'autre. Les offres d'emploi ressemblent davantage à une longue énumération de compétences qu'à un profil type. Cela s'explique évidemment par la multiplicité des domaines touchés par le développement social urbain. Personne n'est susceptible de posséder l'ensemble de ces compétences ni, bien sûr, l'ensemble des connaissances qui sont demandées. Si les employeurs demandent des connaissances tant juridiques que sociales ou urbanistiques... il apparaît clairement que la personne qui est recherchée doit être un généraliste plutôt qu'un spécialiste de l'un de ces domaines. Cela permet également à l'employeur d'avoir une marge de manœuvre plus large quant au recrutement puisque plus de candidats doivent être amenés à se présenter et un choix fait par rapport à une grille de critères sera naturellement plus large. Cependant, depuis que la politique de la ville existe, aucun référentiel de compétences n'a été effectué.

Mais à savoir si l'on va vers une professionnalisation des métiers de la politique de la ville, personne jusqu'ici n'a réellement su trancher. En effet, les indices qui peuvent permettre d'employer le terme de "professionnalisation » seraient l'existence de réseaux, une reconnaissance mutuelle de chacun comme " professionnel », également l'émergence d'une « authentique filière » universitaire qui prépare à exercer ce poste. Or, il est jusqu'ici difficile de repérer une réelle filière universitaire puisque les intitulés des diplômes sont très différents et ils sont rattachés à des UFR (unité de formation et de recherche) qui peuvent être juridique, sociologique, département d'économie ou d'aménagement. Bien qu'originellement très éloigné des affaires sociales ou urbaines, l'Institut d'études européennes de l'Université Paris 8 propose depuis quelques années une spécialité : "villes et nouveaux espaces européens de gouvernance » de master qui peut préparer au métier de chef de projet.

Il existe principalement deux réseaux des professionnels de la politique de la ville : l'inter réseaux DSU que nous avons cité supra et Amadeus. Le premier s'intéresse particulièrement aux questions que nous évoquons ici, à savoir les évolutions et les métiers du développement social urbain. Amadeus est un réseau plus réduit en nombre de membres mais 
très actif sur les questions d'intégration de la politique de la ville aux services municipaux. Il est difficile d'en mesurer le poids sur la question de la professionnalisation. Lorsque nous avons demandé aux acteurs du développement social de Périgueux s'ils se reconnaissent entre professionnels, plusieurs ont répondu qu'il y a une reconnaissance au travers de «leur méthode » de travail. Ils savent qu'ils appartiennent à la même catégorie de professionnels par leur connaissance et leur maîtrise des outils spécifiques à la politique de la ville.

Le rapport Brévan-Picard souhaite voir une cellule de coordination sur la professionnalisation des agents locaux de médiation sociale ainsi que le renforcement des parcours de formation, en particulier pour les personnes bénéficiant du dispositif emploi jeune ${ }^{9}$. La professionnalisation des agents de développement social urbain est un processus en cours mais non encore abouti. Comme le précisent Jean-François Sipp et Maurice Blanc ${ }^{10}$, la question de la professionnalisation doit servir à « professionnaliser des individus pour faciliter leur insertion dans des emplois stables » et non à « professionnaliser des activités précaires ».

Si à l'origine les métiers de la ville étaient prévus comme provisoires, le temps aura prouvé le contraire et la question de la professionnalisation est aujourd'hui un enjeu de taille. Les profils professionnels des acteurs de la politique de la ville ont largement évolués depuis le début des années 1980 mais l'évolution est encore en cours. Nous pouvons former deux hypothèses pour l'avenir : peut-être allons nous vers une stabilisation des statuts et des profils des professionnels de la politique de la ville si le processus de professionnalisation touche un jour à sa fin mais il est tout à fait probable que les métiers du développement social urbain, par leur nature même, restent des métiers « flous » en constante évolution.

\section{Bibliographie indicative :}

- Brevan, C. Picard, P. Une nouvelle ambition pour les villes. De nouvelles frontières pour les métiers. Rapport à Monsieur Claude Bartolone, ministre délégué à la ville. Septembre 2000 .

- Ion, J. Le travail social à l'épreuve du territoire. Paris, Dunod, 2005

- Jeannot, G. Les métiers flous. Travail et action publique. Toulouse, Octarès, 2005

- Sipp, J-F. Blanc, M. Les métiers de la ville et du développement social urbain. Rapport pour la Délégation interministérielle à la ville. Mars 2000.

- Quand la démocratie locale se professionnalise... Actes de la rencontre organisée le 23 octobre 2003, Profession banlieue.

\footnotetext{
${ }^{1}$ Ion, J. Le travail social à l'épreuve du territoire. Paris, Dunod, 2005.

${ }^{2}$ Quand la démocratie locale se professionnalise... Actes de la rencontre organisée le 23 octobre 2003,

Profession banlieue. p. 95 à 148

${ }^{3}$ Série de huit entretiens réalisés du 19 au 22 février 2008 auprès des chargés de mission politique de la ville des communes de Périgueux, Boulazac, Coulounieix-Chamiers, de la Communauté d'Agglomération Périgourdine, du chargé de mission au Conseil Général, au SGAR et à la Préfecture de Département.

${ }^{4}$ Brevan, C. Picard, P. Une nouvelle ambition pour les villes. De nouvelles frontières pour les métiers. Rapport à Monsieur Claude Bartolone, ministre délégué à la ville. Septembre 2000. p. 48-53.

${ }^{5}$ Série de huit entretiens réalisés du 19 au 22 février 2008.

${ }^{6}$ Délégation interministérielle à la ville.

${ }^{7}$ Jeannot, G. Les métiers flous. Travail et action publique. Toulouse, Octarès, 2005.

${ }^{8}$ Offres d'emploi in La Gazette des communes $\mathrm{n}^{\circ} 1922$ du 25/02/2008.

${ }^{9}$ Brevan, C. Picard, P. Une nouvelle ambition pour les villes. De nouvelles frontières pour les métiers. P. 103104.

${ }^{10}$ Sipp, J-F. Blanc, M. Les métiers de la ville et du développement social urbain. Rapport pour la Délégation interministérielle à la ville. Mars 2000. p. 14.
} 